\title{
PHYTOCHEMICL AND ANTIPROLIFERATIVE INVESTIGATIONS OF STRYCHNOS NUX-VOMICA LEAVES
}

\author{
Omayma A. Eldahshan ${ }^{1}$ and Mohamed \\ ${ }^{1}$ Pharmacognosy Department, Faculty of Pharmacy, Ain shams University, Cairo, Egypt \\ ${ }^{2}$ Pharmacology Department, Faculty of Veterinary Medicine, Suez Canal University, \\ Ismailia, Egypt.
}

\begin{abstract}
There are no uses for the strychnine tree (Strychnos nux-vomica L.) in modern medicine although it was widely used in medicine before World War II. The properties of Nux Vomica are those of the alkaloid strychnine. Herein we investigated phytochemically the aqueous methanolic extract of the S. Nux vomica and isolated five phenolic compounds; Kaempferol-7 Glucoside 1, 7 hydroxy Coumarin 2, Quercetin-3-Rhamnoside $\underline{\mathbf{3}}$, Kaempferol 3-rutinoside $\underline{\mathbf{4}}$, and Rutin $\underline{\mathbf{5}}$. At the same time, the cytotoxic activity of the extract was evaluated against different cancer cell lines. The extract showed potential cytotoxic activity against human epidermoid larynx carcinoma cells, with $\mathrm{IC}_{50}$ value $17.8 \mu \mathrm{g} / \mathrm{mL}$. It also exhibited promising cytotoxic activity against breast carcinoma cell line (MCF-7) with $\mathrm{IC}_{50}$ $36.3 \mu \mathrm{g} / \mathrm{mL}$. Colon carcinoma cells were the least one affected by the extract as its $\mathrm{IC}_{50}$ was $41.2 \mu \mathrm{g} / \mathrm{mL}$.
\end{abstract}

\section{INTRODUCTION}

Herbal medicine is still the mainstay of about $75 \%$ to $80 \%$ of the world population, mainly in the developing countries, with the aim of promoting primary health care with better cultural acceptability, human compatibility and fewer side effects. The introduction of medicinal plants in treatment of various diseases is increasing and become now a new trend for discovering new drugs derived from plants (Abdel Daim, 2012). The strychnine tree (Strychnos nux-vomica L.) belonging to Family Loganiaceae has been a very promising drug for certain disorders since long time. There are many works reported regarding the biological activity of $S$. nux-vomica seeds, stem barks and roots related to the alkaloids isolated. Strychnos nux-vomica L. (SN) has been cited in Indian traditional medicine (Ayurveda) for the treatment of paralysis, diabetes, gonorrhea, anemia and bronchitis etc. It has been shown to possess anti-oxidant and anti-snake venom activity (Tripathi, 1998; Tripathi and Chaurasia, 2000; Philippe, et al., 2004). S. nux vomica seeds extract prevents lipid peroxidation by chelating metal ions (Tripathi and Chaurasia, 2000). Since nothing could be traced in literature concerning the phenolic content of leaves of S. nuxvomica and as part of an ongoing study to discover potential bioactive phenolics from terrestrial plant sources (Eldahshan, 2008), the present study was directed to investigate the phenolics present in the aqueous methanol extract obtained from the leaves of S. nux-vomica and to investigate their ability to be used cytotoxic agent(s). 


\section{MATERIAL AND METHODS}

\section{Phytochemical investigation}

Plant source

Strychnos nux-vomica leaves were collected from Zoo Garden in Giza, Egypt, 2011 and were authenticated by Prof. Dr. Abdel Salam El Noyehy, Prof. of Taxonomy, Faculty of Science, Ain Shams University, Cairo, Egypt. Voucher specimen was deposited at the herbarium of Pharmacognosy Department, Faculty of Pharmacy, Ain Shams University, Cairo, Egypt. The leaves were dried in shade and reduced to a fine powder.

\section{Extraction and isolation}

The intact air dried plant material $(1 \mathrm{~kg})$ was comminuted to powder then boiled in distilled water for 2 hours then filtered while hot. The filtrate was completely evaporated in vacuo at $\approx 55^{\circ} \mathrm{C}$ till dryness. The solid residue was then extracted with methanol till exhaustion. The combined methanol extracts was evaporated in vacuo till dryness.

Thirty five grams of the extract were applied on Cellulose column. Elution started with water then water and methanol of decreasing polarity. Six gram of water fraction was chromatographed on polyamide column. Water and water methanol were the solvent system. Fractions 4, 5, 6 and 7 (water eluted) was gathered and applied on paper chromatography using BAW as solvent system. Two compounds were isolated 1 and 2 . Fractions 24-32 (80\%) were pooled and then applied on sephadex column to isolate two compounds $3 \& 4$. Fraction 34 was applied on silica gel column to isolate compound no.5, from solvent system chloroform and methanol.

\section{Chemicals}

Kaempferol, quercetin, apigenin and luteolin were obtained from NMR department, NRC, Cairo. Glucose, rhamnose and glucuronic acid were purchased from E.Merk, Darmstadt Germany. Sephadex LH-20: Phatrmacia fine chemicals, Paper chromatography was carried out on sheets of unwashed Whatman No. 1 paper (Whatman Ltd. Maidstone, Kent, England). Silica gell 60 for column chromatography (E-Merk), particle size (70-230 mesh), precoated silica gel 60 F 254 sheets for TLC (Riedel-De Haem AG, Germany) were also used.

\section{Ultraviolet spectrophotometric analysis}

Chromatographically pure materials $1 \mathrm{mg}$ each were dissolved in analytically pure methanol then subjected to UV spectroscopic investigation in $4 \mathrm{ml}$ capacity quartz cells $1 \mathrm{~cm}$ thick using a Carl Zeiss spectrophotometer PMQ II. $\mathrm{AlCl}_{3}, \mathrm{AlCl}_{3} / \mathrm{HCl}$, fused $\mathrm{NaOAc} /$ $\mathrm{H}_{3} \mathrm{BO}_{3}$ and $\mathrm{NaOMe}$ reagents were separately added to the methanolic solution of investigated material and UV measurements were then carried out.

Nuclear magnetic resonance spectroscopic analysis

The NMR spectra were recorded on a Varian Mercury VX-500 NMR spectrometer. ${ }^{1} \mathrm{H}$ - spectra run at $500 \mathrm{MHz}$ and ${ }^{13} \mathrm{C}$ - spectra were run at $100 \mathrm{MHz}$ in deutrated dimethylsulphoxide (DMSO- $d_{6}$ ). Chemical shifts are quoted in $\delta$ and were related to that of the solvents.

\section{Cytotoxic activity}

All cell lines of a well differentiated carcinoma were obtained from the American Type Culture Collection (ATCC) 
Chemical used Mo., USA)

Dimethyl sulfoxide (DMSO), crystal violet and trypan blue dye (Sigma, St.Louis,

DMEM, RPMI-1640, FBS, HEPES buffer solution, L-glutamine, gentamycin and $0.25 \%$ Trypsin-EDTA (Bio Whittaker ${ }^{\circledR}$ Lonza, Belgium). Crystal violet stain (1\%)

Cytotoxicity evaluation using viability assay

Cell toxicity was monitored by determining the effect of the test sample on cell morphology and cell viability through viability assay (Mosmann, 1993; Vijaya, et al., 2004).

\section{RESULTS AND DISCUSSION}

\section{Identification of compounds 1-5}

Phytochemical investigation of the aqueous methanolic extract of $S$. nux vomica leaves using column fractionation on cellulose, sephadex LH20, silica and paper chromatography resulted in the isolation of 5 compounds: Kaempferol-7 Glucoside $\underline{\mathbf{1}}, 7$ hydroxy Coumarin $\underline{\mathbf{2}}$, Quercetin-3-Rhamnoside $\underline{\mathbf{3}}$, Kaempferol 3-rutinoside $\underline{\mathbf{4}}$, and Rutin $\underline{\mathbf{5}}$. The structures of these compounds were unambiguously determined by their chromatographic behaviors as well as spectroscopic analysis via $\mathrm{UV}$, and ${ }^{1} \mathrm{H}-\mathrm{NMR}$ and ${ }^{13} \mathrm{C}-$ NMR.

\section{Cytotoxic activity}

In vitro cytotoxic assay of the aqueous methanol leaf extract of $S$. nux vomica showed potential cytotoxic activity against human epidermoid larynx carcinoma cells, with $\mathrm{IC}_{50}$ value $17.8 \mu \mathrm{g} / \mathrm{mL}$. It also exhibited promising cytotoxic activity against breast carcinoma cell line (MCF-7) with $\mathrm{IC}_{50} 36.3 \mu \mathrm{g} / \mathrm{mL}$. Colon carcinoma cells were the least one affected by the extract as its $\mathrm{IC}_{50}$ was $41.2 \mu \mathrm{g} / \mathrm{mL}$ (Fig. 2).

\section{Compound 1:}

Kaempferol-7-O- $\beta$-D-glucopyranoside

UV/Vis $(\lambda \max , \mathrm{MeOH}, \mathrm{nm}): 267,320 \mathrm{sh}, 360 .{ }^{\mathbf{1}} \mathbf{H}-\mathbf{N M R}\left(\mathrm{CD}_{3} \mathrm{OD}\right) \delta: 8.25\left(\mathrm{~d}, J=8.8, \mathrm{H}-2^{\prime}\right.$, $\left.6^{\prime}\right), 7.73\left(\mathrm{~d}, J=8.8, \mathrm{H}-3^{\prime}, 5^{\prime}\right), 6.25(\mathrm{~d}, J=2.1), 6.19$ (d, $\left.J=2.1\right), 5.82(\mathrm{~d}, J=9.7$, glc-H-1). 6“). (Chunhua, et al., 2012)

\section{Compound 2:}

Umbelliferone; 7 Hydroxy coumarin

UV/Vis $(\lambda \max , \mathrm{MeOH}, \mathrm{nm}): 216,245,258,279,322 ;{ }^{1} \mathbf{H}-\mathbf{N M R}\left(\mathrm{CD}_{3} \mathrm{OD}\right) \delta: 6.19(1 \mathrm{H}, \mathrm{d}, J$ $=9.5 \mathrm{~Hz}, \mathrm{H}-3), 6.78(1 \mathrm{H}, \mathrm{d}, J=2.3 \mathrm{~Hz}, \mathrm{H}-8), 6.87(1 \mathrm{H}, \mathrm{dd}, J=8.5,2.3 \mathrm{~Hz}, \mathrm{H}-6), 7.46(1 \mathrm{H}$, $\mathrm{d}, J=8.5 \mathrm{~Hz}, \mathrm{H}-5), 7.86(1 \mathrm{H}, \mathrm{d}, J=9.5 \mathrm{~Hz}, \mathrm{H}-4)(\mathrm{Kim}$, et al., 2006)

\section{Compound $\underline{3}$ :}

Quercetin 3-O- ${ }^{1} \mathrm{C}_{4}$-L-rhamnoside ; Quercitrin 
UV/Vis $(\lambda \max , \mathrm{MeOH}, \mathrm{nm}) 259,297 \mathrm{sh}, 348 .{ }^{\mathbf{1}} \mathbf{H}-\mathbf{N M R}$ (DMSO- $\left.d 6\right) 0.9$ (d, $J=6 \mathrm{~Hz}, \mathrm{CH}_{3}$ ), $5.2\left(\mathrm{~d}, J=1.5 \mathrm{~Hz}, \mathrm{H}-{ }^{\prime}{ }^{\prime}\right), 6.15(\mathrm{~d}, J=2.2 \mathrm{~Hz}, \mathrm{H}-6), 6.36(\mathrm{~d}, J=2.2 \mathrm{~Hz}, \mathrm{H}-8), 6.95(\mathrm{~d}, J=8.2$ Hz, H-5 ) $7.25\left(\mathrm{dd}, J=2.2 \mathrm{~Hz}\right.$ and $\left.J=8.2 \mathrm{~Hz}, \mathrm{H}-6{ }^{\prime}\right), 7.35\left(\mathrm{~d}, J=2.2 \mathrm{~Hz}, \mathrm{H}-2^{\prime}\right),{ }^{13} \mathbf{C N M R}$ (DMSO-d6) 17.67(C-6“), 70.21(C-5“), 70.73(C- 3“), 70.50(C-2“) , 71.35(C-4“), 93.97(C8), 99.20(C-6), 101.91(C-1“'), 103.79(C-10), 115.68(C-2`), 115.78(C-5'), 120.79(C-1'), 121.21(C-6), 134.24(C-3), 145.44(C-3`), 148.78(C-4), $156.66(\mathrm{C}-2), \quad 157.25(\mathrm{C}-9)$, 161.35(C-5), 165.59(C-7), 177.72(C-4).(Omayma, et al., 2011)

\section{Compound 4:}

kaempferol-3-O- $\alpha$-L-rhamnopyranosyl-( $1 \rightarrow 6)-\beta-D$-glucopyranoside;Kaempferol-3-Orutinoside

UV/Vis $(\lambda \max , \mathrm{MeOH}, \mathrm{nm}): 266,350 \mathrm{~nm} .{ }^{\mathbf{1}} \mathbf{H}$ NMR $\left(\mathrm{CD}_{3} \mathrm{OD}\right) \delta: 6.20(\mathrm{~d}, J=1.9 \mathrm{~Hz}, \mathrm{H}-6)$, $6.39(1 \mathrm{H}, \mathrm{d}, J=1.9 \mathrm{~Hz}, \mathrm{H}-8), 6.88$ (d, $\left.J=8.8, \mathrm{H}^{-} 3^{`}, \mathrm{H}^{-} 5^{`}\right), 8.05\left(2 \mathrm{H}, \mathrm{d}, J=8.8, \mathrm{H}-2^{`}, \mathrm{H}^{-}\right.$ $\left.6 `), 5.12\left(\mathrm{~d}, J=7.4, \mathrm{H}-1^{\prime \prime}\right) ; 1.10\left(\mathrm{~d}, J=6.1 \mathrm{~Hz}, \mathrm{H}-6^{\cdots}{ }^{\prime}\right), 4.51\left(\mathrm{~d}, J=1.5 \mathrm{~Hz}, \mathrm{H}^{\prime}\right)^{\prime}\right)$.

(Nabry, et al., 1970)

\section{Compound 5:}

Quercetin-3-O- $\alpha$-L-rhamnopyranosyl-(6“ $\left.\longrightarrow 1^{\prime \prime}\right)-\beta$-D-glucopyranoside; Quercetin 3-Orutinoside; Rutin.

UV/Vis ( $\lambda \max , \mathrm{MeOH}, \mathrm{nm}): 256,266 \mathrm{sh}, 300 \mathrm{sh}, 360 .{ }^{1} \mathbf{H}-\mathbf{N M R}$ (DMSO- $\left.d 6\right)$ 6.21(d, $J=2, \mathrm{H}-$ 6), 6.40 (d, $J=2, \mathrm{H}-8), 7.55$ (d, $\left.J=2.1, \mathrm{H}-2{ }^{\prime}\right), 6.86$ (d $\left.J=9, \mathrm{H}-5^{\prime}\right), 7.56$ (dd, $J=9,2.1, \mathrm{H}-6$ ) ), 5.35 $\left(\mathrm{d}, J=7.4, \mathrm{H}-1^{\prime \prime}\right), 5.12\left(\mathrm{~d}, J=1.9, \mathrm{H}-1^{\prime \cdots}\right), 1.00\left(\mathrm{~d}, J=6.1, \mathrm{CH}_{3}\right),{ }^{13} \mathbf{C}$ NMR (DMSO-d6) $\delta$ 157.3(C-2), 134.1 (C-3), 178.2 (C-4), 157.5 (C-5), 99.5 (C-6), 164.9 (C-7), 94.5 (C-8), 162.1 (C-9), 104.8 (C-10), 122.5 (C-1'), 116.1(C-2`), 145.6 (C-3`), 149.3 (C-4), 117.1 (C-5`), 122.0 (C-6`),101.6 (C-1“), 74.9 (C-2“), 77.3 (C-3“), 72.7 (C-4“), 76.7 (C-5“), 67.9 (C-

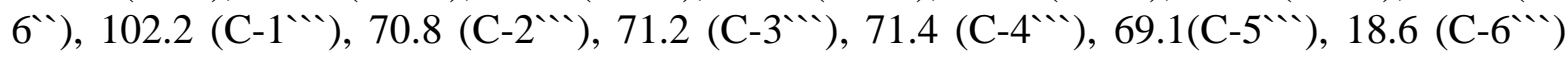
(Fatemeh, et al., 2006). 


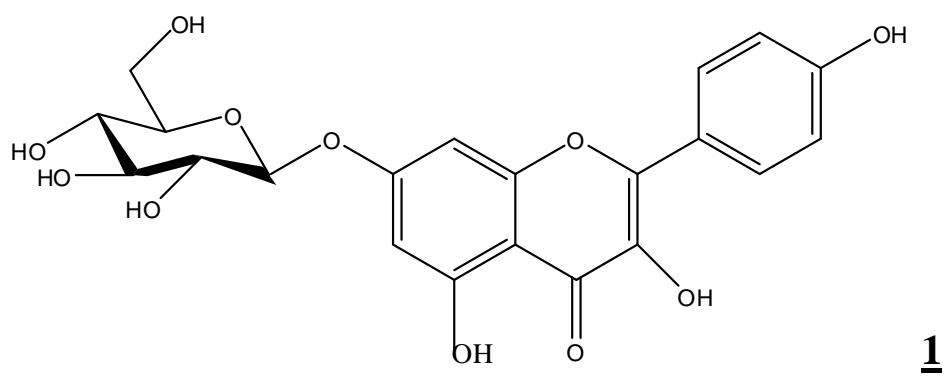

(N)
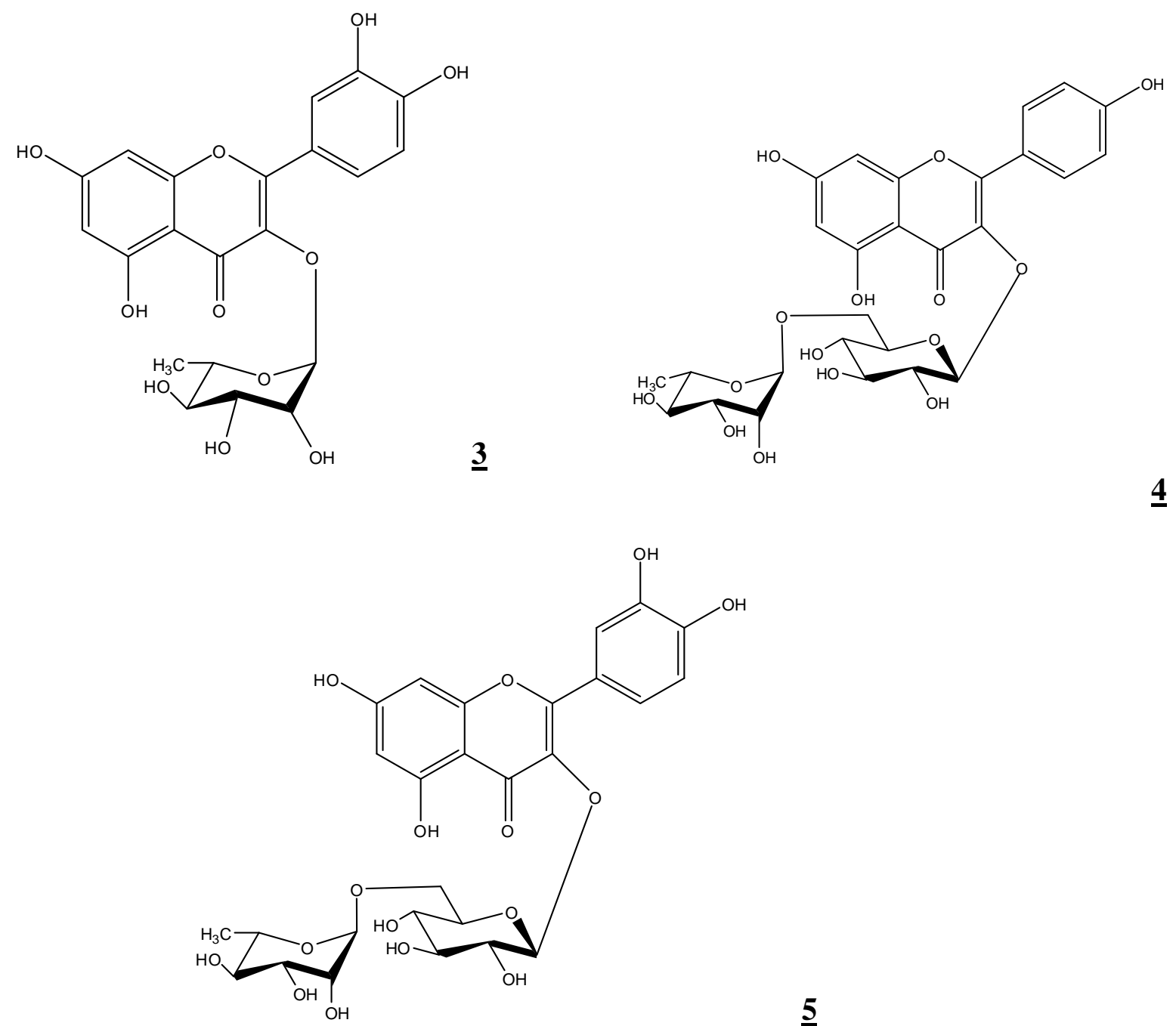

Fig. 1: Compounds isolated from S. nux vomica Leaves 
Hep-2

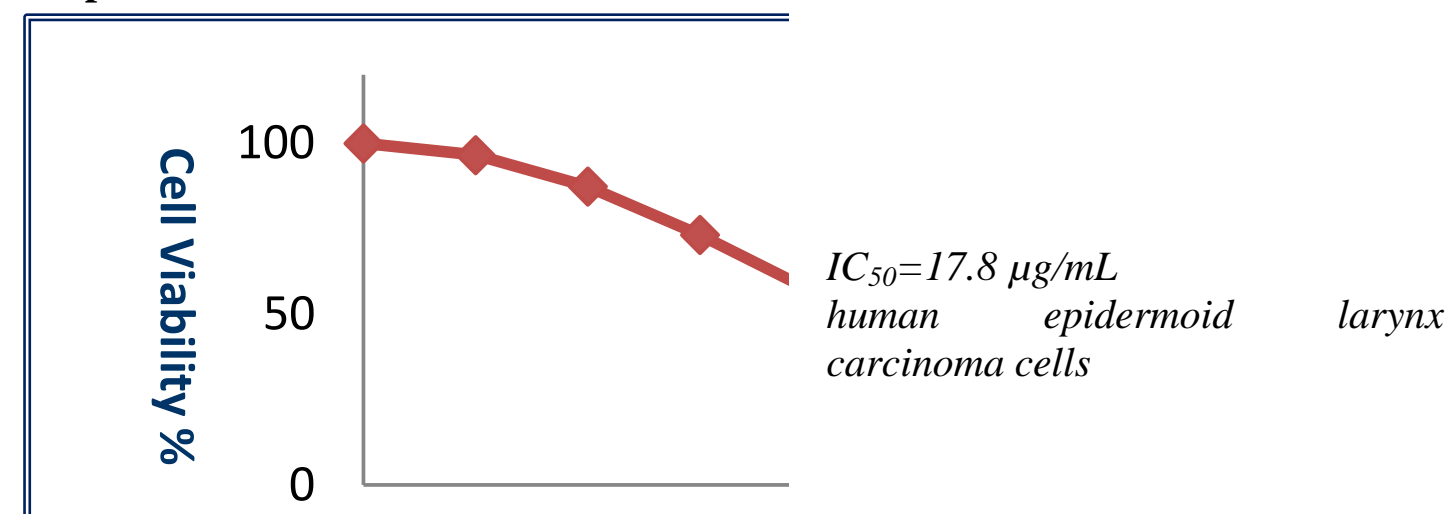

MCF-7

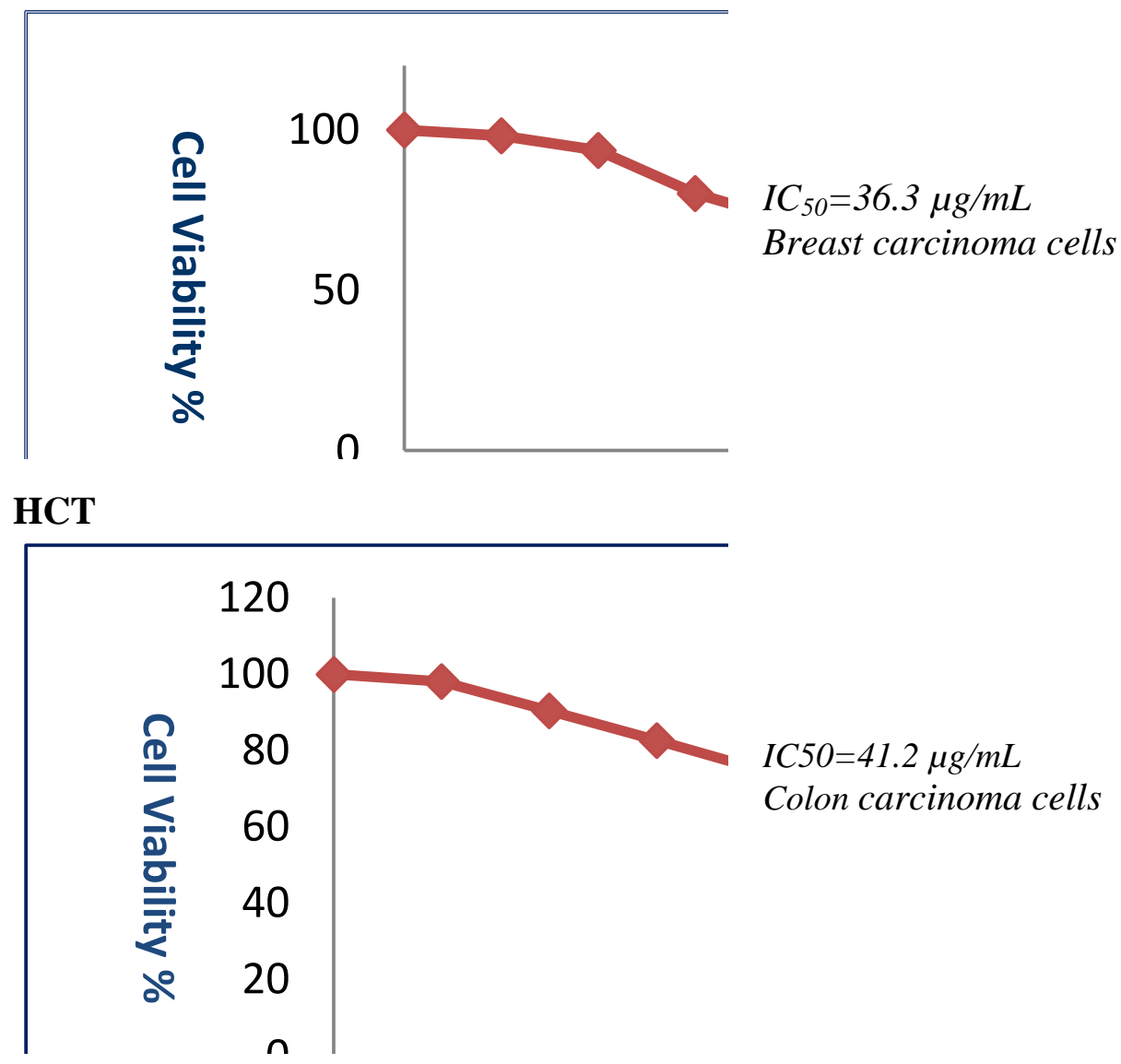

Fig. 2: Cytotoxic activities of S. nux vomica leaves extract

\section{CONCLUSION}

Phytochemical investigation of $S$. nux vomica leaf extract led to the isolation and characterization of five compounds. Apotent cytotoxic activity was detected against human epidermoid larynx carcinoma cells, with $\mathrm{IC}_{50}$ value $17.8 \mu \mathrm{g} / \mathrm{mL}$, followed by breast carcinoma cell line (MCF-7) and then colon carcinoma cells which was the least one affected by the extract. The current study indicates that the phenolic compounds isolated from $S$. nux vomica leaf are promising molecules with potentially useful cytotoxic activity profiles. This confirms that leaves of this plant have a great value as a source of compounds with pharmaceutical applications. 


\section{ACKNOWLEDGEMENT}

The authors acknowledge Prof. Dr Abdel Salam El Noyehy, Prof. of Taxonomy, Faculty of Science, Ain Shams University, Cairo, Egypt for authentication the leaves of Strychnus nux vomiva. The authors are also grateful for The Regional Center for Mycology and Biotechnology, Al-Azhar University, Cairo, Egypt for hosting the cytotoxic activities.

\section{Conflict of interest statement}

All authors declare that there is no conflict of interest $t$ relevant to this study.

\section{REFERENCES}

Abdel Daim M. (2012): Antinociciptive effect of ginger extract in streptozotocin-induced diabetic rats Assiut Vet. Med. J. 58(132):317-327.

Tripathi, Y.B., (1998): Free radicals in Ayurveda. Ancient Sci. Life 17, 158-168.

Tripathi, Y.B., Chaurasia, S., (2000): Interaction of Strychnos nux-vomica products and iron: with reference to lipid peroxidation. Phytomedicine 7, 523-528.

Philippe, G., Angenot, L., Tits, M., Frederich, M., (2004): About the toxicity of some Strychnos species and their alkaloids. Toxicon 44, 405-416.

Omayma A. Eldahshan, Nahla A. Ayoub, Abdel-Nasser B. Singab and Mohamed M. AlAzizi, (2008): Potential Superoxide Anion Radical Scavenging Activity of Doum Palm (Hyphaene thebaica L.) Leaves Extract, Rec. Nat. Prod. 2:3, 83-93.

Mosmann, T. (1993): Rapid colorimetric assay for cellular growth and survival:application to proliferation and cytotoxicity assays. J Immunol. Methods, 65, 55-63.

Vijaya, P., Raghu, C., Ashok, G., Dhanaraj, S.A. and Suresh, B. (2004): Antiviral activity of medicinal plants of Nilgiris. Indian J Med. Res, 120, 24-29.

Chunhua Lu1, Yaoyao Li1, Liji Li2, Lanying Liang3, Yuemao Shen1, (2012): Antiinflammatory activities of fractions from Geranium nepalense and related polyphenols, *Drug Discoveries \& Therapeutics. 6(4):194-197.

Kim, J.S.; Kim, J.C.; Shim, S.H.; Lee, E.J.; Bae, W.J.K.; Son, K.H.; Kim, H.P.; Kang, S.S.; Wook, H. (2006): Chemical constituents of the root of Dystaenia takeshimana and and their anti-inflammatory activity. Arch. Pharma. Res., 29, 617-623.

Omayma A. Eldahshan, (2011): Isolation and Structure Elucidation of Phenolic Compounds of Carob Leaves Grown in Egypt, Current Research Journal of Biological Sciences 3(1): 52-55.

Mabry, T.J., Markham, K.R., Thomas, M.B., (1970): The Systematic Identification of Flavonoids. Springer-Verlag, New York, Heidlberg, Berlin, pp. 354.

Fatemeh F., Abbas D., Roya A. and Satyajit D. Sarker (2006): Extraction of Flavonoids and Quantification of Rutin from waste Tobacco Leaves. Iranian Journal of Pharmaceutical Research, 3: 222-227. 
در اسة كيميائية عقاقيرية على أوراق نبات الجوز المقىع ودراسة مدى تأثيرها على تكاثر الخلايا السرطانية

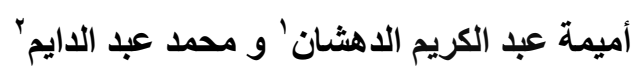

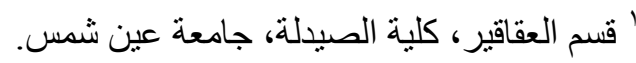

كَّم الادوية و السموم، كلية طب بيطرى، جامعة قناة السويس، الاسماعيلية.

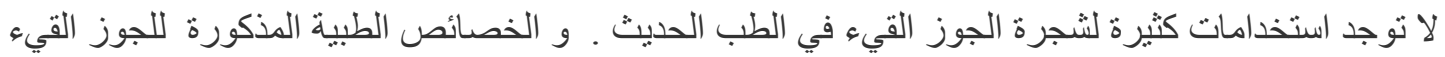

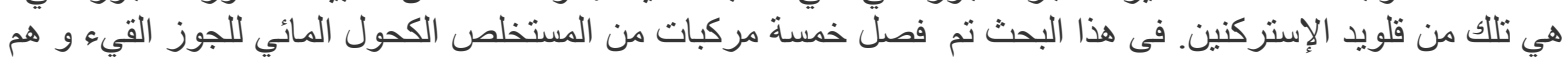

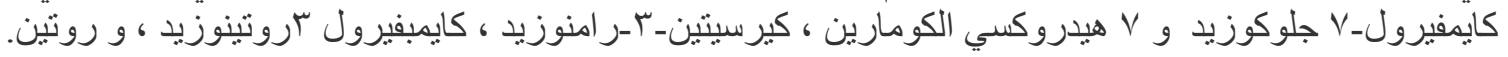

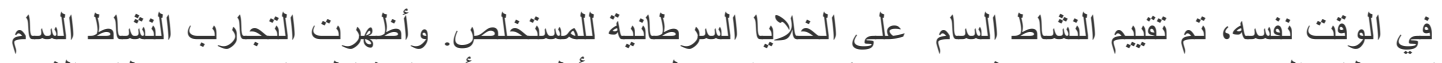

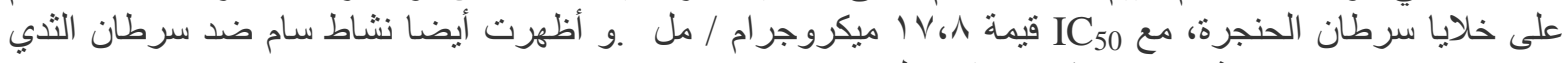

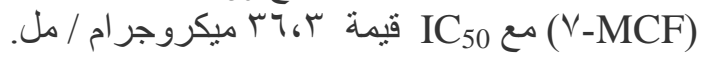

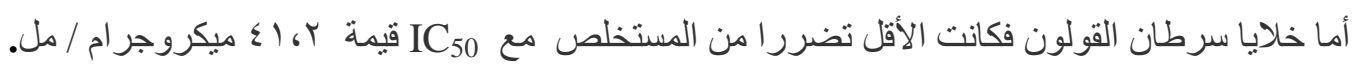

